\title{
Estudios comparativos para la determinación de Ocratoxina A en granos de café verde
}

\begin{abstract}
Carlos Vallejos"
* Profesor e investigador de la Facultad de Ciencia, Tecnología y Ambiente de la Universidad Centroamericana (UCA). Apartado 69. Managua, Nicaragua. E-mail: carlosv@ns.uca.edu.ni
\end{abstract}

Recibido: abril 2005 / Aceptado: diciembre 2005

LA OCRATOXINA A (OTA) ES UNA DE LAS MICOTOXINAS MÁS NEFROTÓXICA y nefrocancerígena producida por varias especies de Aspergillus y Penicillium. OTA se encuentra frecuentemente en productos de origen vegetal como cereales, cerveza, vino, granos de café, cacahuetes y frutas secas. Desde que se ha descrito su presencia en el café verde, las compañías de café han prestado mucha atención a la calidad micotóxica del café verde para garantizar la seguridad alimentaria. En este estudio se comparan tres métodos para la determinación de OTA: Cromatografía de capa fina-TLC, Cromatografía líquida de alta resolución-HPLC y Vicam Ochratest en muestras de café, y se ha validado el Vicam Ochratest para el análisis de OTA en el café con columnas de inmunoafinidad. Por su bajo costo y la similitud de los resultados comparados con el método HPLC, el uso del método Vicam Ochratest se presenta como muy útil para los pequeños productores de café de Nicaragua, pues les permitirá mejorar su competitividad en el mercado internacional.

Palabras clave: café-análisis / control de alimentos / micotoxinas / nefrología

\section{Introducción}

La Ocratoxina A (OTA) es una micotoxina de origen fúngico, producida principalmente por Penicillum verrucosum en climas templados y por varias especies de Aspergillus en productos de climas tropicales y subtropicales. La especie más conocida de Aspergillus productora de OTA es A. ochraceus, pero existen también otras: A. sulphureus, A. ostanius y A. sclerotiorum. De los Aspergillus, solo el A. ochraceus y el A. ostanius se señalan como fuentes importantes de OTA en los cereales, cacao, té y café.

La OTA tiene un esqueleto pentaquetido (M. Frank, 1999) y contiene una porción de isocumarina clorado ligado a través de un grupo de carboxílico a la L-fenilalanina mediante un enlace amido (v. Ilustración 1): 


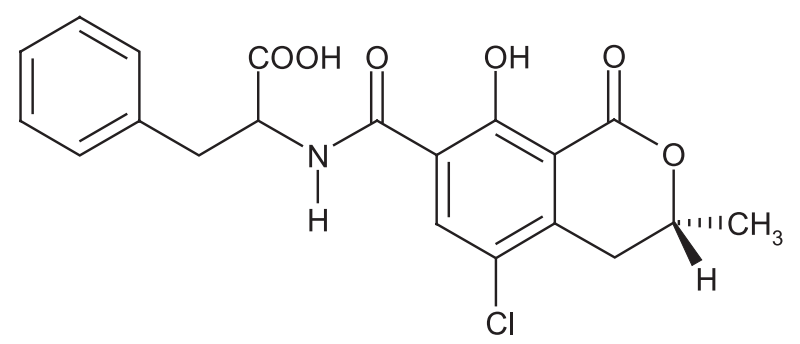

Ilustración 1. Estructura de la Ocratoxina A (OTA)

OTA forma parte de una familia de micotoxinas que comprende las ocratoxinas B y C, que son el análogo declorado y el éster etílico, respectivamente. El ácido carboxílico de isocumarina (ocratoxina $\alpha$ ) y su análogo declorado (ocratoxina $\beta$ ) pueden también detectarse en cultivos de cepas de Aspergillus y Penicillium productoras de OTA y también se han encontrado 4-hidroxi en cultivos de Aspergillus ochraceus.

La contaminación con OTA se da principalmente en productos alimenticios de origen vegetal, como los cereales, algunas legumbres, granos de café, cacao, nueces, cerveza y pan, colonizados previamente por los hongos productores que contaminan los productos en las etapas posteriores de almacenamiento o elaboración. También se ha detectado OTA en productos cárnicos provenientes de animales alimentados con comida contaminada. Los efectos tóxicos de las micotoxinas son muy diversos y muchas desarrollan varias acciones tóxicas diferentes. Entre las acciones más patógenas están: lesiones hepáticas, trastornos renales, carcinogénesis, efectos neurotóxicos, acciones alérgicas, irritación de mucosas y fotosensibilización cutánea. Estos efectos se pueden producir mediante la ingestión de pequeñas cantidades de estas sustancias.

El desarrollo de los hongos y la producción de micotoxinas requieren de ciertas condiciones ambientales (L. R. Batista et al., 2003):

Factores físicos: Humedad, temperatura, zonas de microflora (pequeñas zonas del alimento con alto contenido de humedad) e integridad física del grano o del alimento.

Agua disponible: Merece especial mención el concepto de actividad de agua o el agua disponible, normalmente designada como $\mathrm{a}_{\mathrm{w}}$ La $\mathrm{a}_{\mathrm{w}}$ (actividad de agua) indica la cantidad de agua disponible para el desarrollo de los microorganismos, una vez alcanzado el equilibrio hídrico en el sistema alimento/medio ambiente. La $\mathrm{a}_{\mathrm{w}}$ se refiere a la relación entre la presión de vapor del agua en el substrato $(\mathrm{P})$ y del agua pura $(\mathrm{Po})$, a la misma temperatura, $\left(\mathrm{a}_{\mathrm{w}}=\mathrm{P} /\right.$ Po). El agua pura tiene una $a_{w}=1$. En los alimentos, la aw será siempre inferior a 1. La mayor parte de los hongos que contaminan los cereales necesitan valores superiores a 0.7 .

Factores químicos: Composición del substrato, pH, nutrientes, minerales y disponibilidad de oxígeno.

Factores biológicos: Presencia de invertebrados y cepas específicas. Los insectos actúan como agentes de diseminación de la microflora y contribuyen al crecimiento y multiplicación 
de los hongos. El propio metabolismo de los insectos eleva el contenido de humedad del sustrato y la rotura del pericarpio permite la infección del interior del grano.

Cepas específicas: En una misma especie fúngica, no todas las cepas se comportan de la misma forma. En una especie fúngica hay estirpes productoras de micotoxinas y otras que son incapaces de producirlas.

En la Tercera Conferencia Internacional sobre Micotoxinas, organizada por la Organización para Agricultura y Alimentación (FAO), Organización Mundial de la Salud (OMS) y Programa de las Naciones Unidas para el Medio Ambiente (PNUMA) en Túnez, 1999 se presentó un estudio sobre el sistema de análisis de puntos críticos y control de la contaminación de OTA durante la producción del café (M. Frank, 1999). Se examinaron cadenas de producción de seis naciones de tres continentes (Brasil, Etiopía, India, Indonesia, Kenia y Venezuela) para conocer la diversidad de los procedimientos de producción, como elemento necesario para elaborar un modelo preciso de los procedimientos de producción de café.

La contaminación por micotoxinas de productos expuestos se produce como resultado de las condiciones ambientales en el campo o de operaciones inadecuadas durante la recolección, el almacenamiento y la elaboración. Los programas de Análisis de Peligros y de Puntos Críticos de Control (APPCC) han sido útiles para hacer frente a los riesgos asociados con la posible contaminación de productos alimenticios. Los programas de inocuidad de los alimentos suelen utilizar información sobre los factores que propician la contaminación para establecer medidas preventivas y de control con el objetivo de producir alimentos inocuos y sanos. Al introducir un programa eficaz de APPCC para las micotoxinas, se determinan los principales elementos que pueden utilizarse o modificarse para reducir la formación de micotoxinas en el campo y en el lugar de almacenamiento; por ejemplo, limitar la infestación por insectos y vigilar del nivel de humedad de los productos. Determinados procedimientos de elaboración y descontaminación pueden contribuir a reducir el nivel de las micotoxinas mediante la separación física de las almendras, granos o nueces dañadas, inmaduras e infestadas por mohos, y la inactivación física y química y/o la eliminación de la toxina. Para elaborar y aplicar los programas de inocuidad de los alimentos basados en el sistema de APPCC son necesarios conocimientos técnicos en diversos ámbitos. Por eso, la FAO se ha esforzado en dar asistencia técnica a sus Estados Miembro con el fin de fortalecer la capacidad nacional para introducir y mantener programas eficaces de gestión de micotoxinas, basados en el sistema de APPCC.

Se conocen los siguientes métodos para determinar la presencia de OTA en el café: TLC, HPLC con columnas de inmunoafinidad y test rápido Ochratest. Estos métodos se aplicaron en este trabajo de investigación a fin de evaluar un método eficaz, sensible, de amplio alcance y bajo costo, accesible a los pequeños caficultores del Norte de Nicaragua, para mejorar la calidad de su café, con miras a alcanzar un mejor precio en el mercado internacional.

Respecto a la calidad del café, la Unión Europea propone que todo el café debe ser obligatoriamente muestreado, establece un límite máximo de 3 ppb en muestras (Reglamento de la Comisión Europea, No. 2377/90) y define un plan de vigilancia que obliga a terceros países al chequeo físico de los productos destinados a la exportación (Reglamento CE No. 
466/2001 y Comisión Europea No. 123/2005).

Tomando en cuenta estas recomendaciones, este trabajo pretende determinar la presencia de OTA por los métodos TLC, HPLC-columnas de Inmunoafinidad y Vicam Ochratest; comparar los métodos en base a criterios analíticos; y validar el método seleccionado en base a un protocolo de validación.

\section{Materiales y métodos}

En la parte experimental de este trabajo, se comparan los métodos de TLC, HPLC-Columnas de inmunoafinidad y el método rápido de laboratorio Vicam Ochratest para validar un método confiable, preciso, exacto y de bajo costo.

Para determinar la presencia de OTA en los granos de café por cromatografía de capa fina, se siguió el procedimiento descrito por Pittet y Royer (2002). Para ello, se adaptó la información a fin de calcular las concentración de adiciones y de trabajo a partir de la solución madre de OTA.

La solución estándar de OTA (10 $\mu \mathrm{g} / \mathrm{mL})$ se preparó diluyendo la solución estándar de OTA (50 $\mu \mathrm{g} / \mathrm{mL})$ cinco veces con tolueno/ácido acético (99:1). La solución de adición preparada se colocó en un freezer oscuro a- $18^{\circ} \mathrm{C}$, Después, la solución estándar de trabajo a $0.5 \mathrm{ng} / \mathrm{mL}$ se preparó transfiriendo $500 \mu \mathrm{L}$ de la solución de $10 \mu \mathrm{g} / \mathrm{mL}$ a un Erlenmeyer de $10 \mathrm{~mL}$ y diluyendo el volumen deseado con acetonitrilo/tolueno/ácido acético (50:90:41). Esa solución se colocó en el refrigerador a $4^{\circ} \mathrm{C}$.

Para la extracción, se tomó una porción de 50 granos de café verde que se depositó en un Erlenmeyer de $500 \mathrm{~mL}$. La muestra se trató con $25 \mathrm{~mL}$ de ácido fosfórico (preparado diluyendo 5.75 gramos de ácido fosfórico al $85 \%$ a $500 \mathrm{~mL}$ de agua), y extraído con $125 \mathrm{~mL}$ de diclorometano, agitando por 60 minutos en un agitador mecánico; posteriormente, se filtró la suspensión resultante.

Posteriormente, alícuotas de $60 \mu \mathrm{L}$ de extracto de muestras de tres gramos de café verde y solución estándar de trabajo de OTA $(0.5 \mathrm{ng} / \mathrm{mL})$ se depositaron en placas de cromatografía de capa de silicagel 60 con una jeringa graduada en microlitros, a lo largo de una línea virtual situada a 100 milímetros de la parte inferior de la placa. Las gotas se aplicaron a 13 milímetros de intervalos sobre la línea.

Durante la aplicación se tuvo cuidado de secar las gotas con una corriente de aire frío para que no excedieran nunca un diámetro de cinco milímetros. La placa cromatográfica se reveló primeramente, con éter dietílico (tanque saturado), aire seco, examinada con luz ultravioleta y cortada aproximadamente un cm arriba de la línea marcada para remover las sustancias que pudieran causar interferencias cuando migran con el solvente. Después, la placa se reveló en la dirección opuesta (cortar abajo), en tolueno/etil acetato/ácido fórmico (5:4:1), de nuevo en tanque saturado, hasta que el frente del solvente alcanzó cinco milímetros de la parte superior de la placa. Después de ese segundo revelado, la placa cromatográfica se secó al aire a temperatura ambiente y se observó visualmente a una longitud de onda de $366 \mathrm{~nm}$ 
en la lámpara ultravioleta. La concentración de OTA en muestras de café verde se determinó en el orden de mayor, igual o menor que $10 \mu \mathrm{g} / \mathrm{kg}$, comparando la intensidad fluorescente de OTA adicionada (con un $\mathrm{R}_{\mathrm{f}}$ de aproximadamente 0.70 ), con el estándar puro de OTA. Esta lectura de la concentración se hizo con una lámpara UV Camag de longitud de onda de 366 $\mathrm{nm}$. A esta longitud de onda, la OTA fluoresce en el rango de las concentraciones aplicadas sobre la placa cromatográfica previamente tratada con indicador de fluorescencia, que no interfiere con el método de detección.

Para la determinación de OTA en granos de café verde por el método HPLC con columnas de inmunoafinidad, se siguió el siguiente procedimiento propuesto por C. Vallejos et. al., (2003) y por E. Dos Santos et. al., (2004):

En la extracción, se tomaron 25 gramos de café verde, se colocaron en un frasco de $500 \mathrm{~mL}$ y se le añadieron $200 \mathrm{~mL}$ de metanol con $\mathrm{NaHCO}_{3}$ al 3\%; luego, se agitó fuertemente por cinco minutos y se filtró a través de papel filtro Whatman No. 4; por último, se tomaron cuatro mL del filtrado y se aforaron a $100 \mathrm{~mL}$ con PBS (solución salina de Buffer de fosfato).

En la Purificación con columnas de inmunoafinidad, se pasaron $100 \mathrm{~mL}$ del extracto de muestra diluido a través de las columnas de inmunoafinidad en un flujo de $2-3 \mathrm{~mL} / \mathrm{min}$ (con presión de vacío controlada). No se permitió que la columna se secara, se lavó la columna con $10 \mathrm{~mL}$ de agua destilada a un flujo de tres $\mathrm{mL} / \mathrm{min}$, se secó la columna aplicando vacío por un tiempo de 30 segundos, se eluyó OTA con cuatro $\mathrm{mL}$ de metanol y se esperó por tres min para permitir que el metanol entrara en contacto con el gel antes de la elusión. Finalmente, se evaporó el eluato hasta sequedad con corriente de nitrógeno a $40^{\circ} \mathrm{C}$ y redisuelvió el residuo en $200 \mu \mathrm{L}$ de fase móvil, mezclando bien e inyectando en el sistema HPLC a lo largo de ocho calibraciones de standards de OTA preparadas en la fase móvil.

\section{A continuación se indican las condiciones cromatográficas para el HPLC:}

Columna: ODS Hypersill $5 \mu \mathrm{m}, 250 \times 4.6 \mathrm{~mm}$ (o equivalente)

Fase móvil: 35\% acetonitrilo, 35\% metanol, 30\% de ácido acético glacial (29:1)

Flujo: $0.8 \mathrm{~mL} / \mathrm{min}$

Temperatura: ambiente

Volumen de inyección: $20 \mu \mathrm{L}$

Detección: Fluorescencia, excitación 332 nm, emisión 476 nm

Para trazar la curva de calibración, se trabajó con las siguientes concentraciones de OTA:

$3.26 \mathrm{E}-01 \mu \mathrm{g} / \mathrm{L}, 6.51 \mathrm{E}-01 \mu / \mathrm{L}, 1.30 \mathrm{E}+00 \mu \mathrm{g} / \mathrm{L}, 2.60 \mathrm{E}+00 \mu \mathrm{g} / \mathrm{L}, 5.21 \mathrm{E}+00 \mu \mathrm{g} / \mathrm{L}$,

$1.04 \mathrm{E}+01 \mu \mathrm{g} / \mathrm{L}, 2.08 \mathrm{E}+01 \mu \mathrm{g} / \mathrm{L}$ y $4.17 \mathrm{E}+01 \mu \mathrm{g} / \mathrm{L}$

Para determinar la presencia de OTA utilizando el test rápido Vicam Ochratest con columnas de inmunoafinidad, se siguió un método de extracción similar al método de extracción por HPLC, con la diferencia que se aplicó otro eluyente. Se siguió el siguiente procedimiento:

En la extracción, se pesaron 25 gramos de café verde y se colocaron en una jarra batidora, se añadieron $50 \mathrm{~mL}$ de metanol: $1 \% \mathrm{NaHCO}_{3}$ (70:30 en volumen), se tapó la jarra metálica, 
se agitó a alta velocidad durante un minuto y luego se filtró. El filtrado se colocó en un recipiente limpio. Finalmente, se pipetearon $10 \mathrm{~mL}$ del filtrado en un recipiente limpio, se diluyeron con $40 \mathrm{~mL}$ de PBS/2\% Tween-20 Wash Buffer y el resultante se mezcló bien.

Para Purificación en las columnas de inmunoafinidad, se dejó pasar a través de la columna Ochratest $10 \mathrm{~mL}$ (equivalente a un gramo de muestra) a una velocidad de una a dos gotas por segundo, hasta que el aire pasase a través de la columna; se dejaron pasar $10 \mathrm{~mL}$ de PBS/2\%Tween-20 Wash Buffer a una velocidad de una a dos gotas por segundo y se pasó aire por 30 segundos. Luego, se dejaron pasar $10 \mathrm{~mL}$ de agua purificada a través de la columna, y se eluyó la columna pasando $1.5 \mathrm{~mL}$ de solución Eluyente Ochratest a una velocidad de una gota por segundo. El eluato se recogió en un tubo de ensayo. Se puso en contacto el eluyente con la columna por tres minutos antes de la elución. Al final, se mezcló todo bien, se colocó la cubeta inmediatamente en el Fluorímetro series-4 previamente calibrado y se leyó la concentración de OTA después de 60 segundos.

En el caso de que se apruebe una legislación sobre la Validación del método analítico, el límite máximo de OTA permitido en el café sería de tres $\mu \mathrm{g} / \mathrm{kg}$ en la muestra. En el Reglamento de la Unión Europea No. 466/2001, se establece este límite y es válido a partir de marzo de 2002.

Para validar un método, se sigue un protocolo de validación (Castro Cells, et.al., 2000). Los aspectos relevantes considerados fueron los siguientes: Linealidad, Exactitud, Precisión y Porcentaje de recuperación.

\section{Resultados}

El método propuesto para la determinación de OTA por TLC brinda información cualitativa de la presencia de OTA en granos de café verde; es decir, la determinación de la presencia o ausencia de OTA a una concentración dada de $10 \mu \mathrm{g} / \mathrm{kg}$.

Las muestras analizadas procedentes de diferentes lugares de Nicaragua están en el rango descrito anteriormente. Los resultados se detallan en el Cuadro 1: 
Cuadro 1. Determinación de OTA vía TLC en granos de café verde procedente de diferentes sitios de Nicaragua

\begin{tabular}{|c|c|}
\hline Origen de la muestra & Concentración de OTA $[\mu \mathrm{g} / \mathrm{L}]$ \\
\hline ISRAEL 1 & $>10 \mathrm{ppb} \odot$ \\
\hline ISRAEL 2 & $>10 \mathrm{ppb} \bullet$ \\
\hline CANAVALIA & $<10 \mathrm{ppb}$ \\
\hline GUAPOTAL & $>10 \mathrm{ppb}$ \\
\hline TUMA & $<10 \mathrm{ppb}$ \\
\hline WASLALA & $<10 \mathrm{ppb}$ \\
\hline DALIA & $<10 \mathrm{ppb}$ \\
\hline RANCHO GRANDE & $<10 \mathrm{ppb}$ \\
\hline NATURALMENTE CONTAMINADA & $>10 \mathrm{ppb}$ 踑 \\
\hline
\end{tabular}

- Café vendido en grano en mercado capitalino

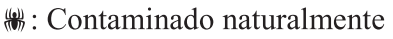

Se puede observar que las muestras de café con mayor contenido de OTA corresponden a café de mala calidad, vendido y/o almacenado en condiciones inadecuadas.

En la determinación cromatográfica por HPLC, la curva de calibración correspondiente a las muestras de patrón inyectadas con su respectiva área se presenta a continuación:

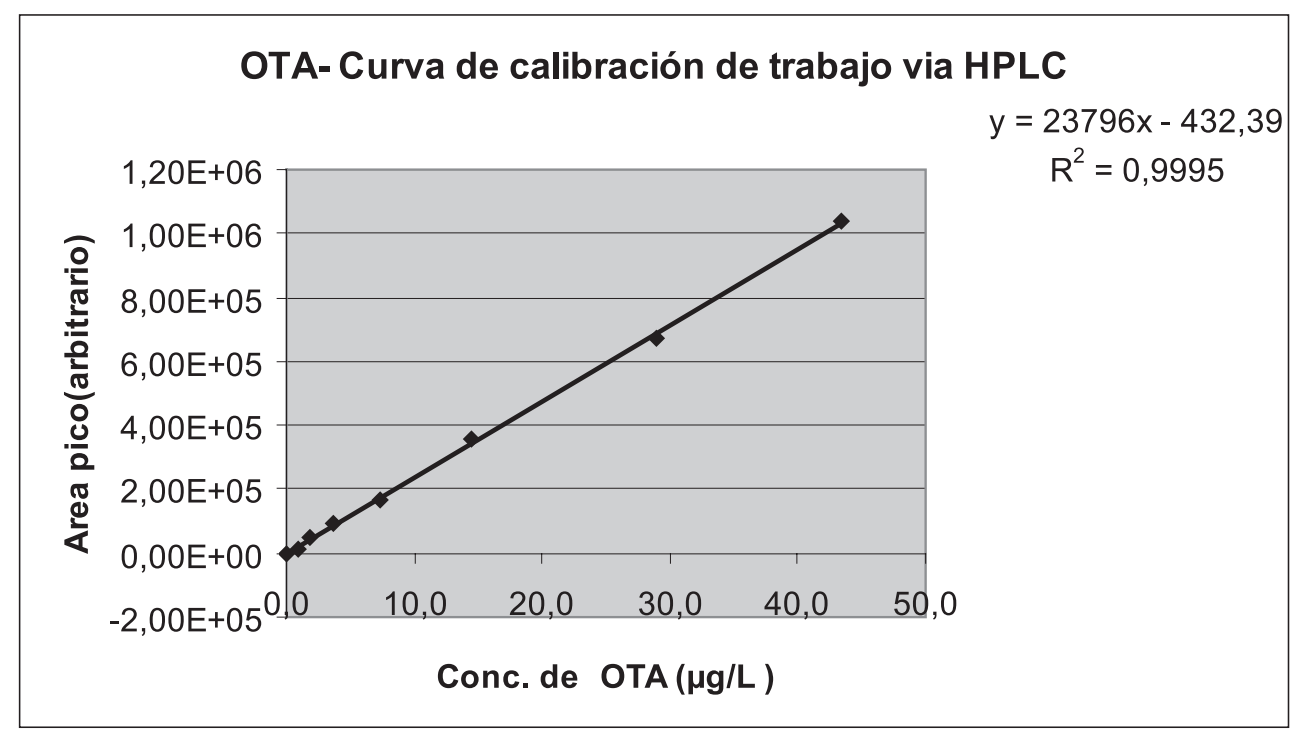

Ilustración 2. Curva de calibración para estándares vía HPLC 
En la Ilustración 2, se observa que el coeficiente de correlación es aceptable. A partir de esta curva de calibración, se calculó la concentración de OTA en muestras de café verde recolectadas en Managua y en la región de Matagalpa.

Los cromatogramas correspondientes a la detección de OTA en los patrones y en una muestra de café, y las concentraciones de OTA para la muestras de los diferentes sitios de Nicaragua se presentan a continuación.

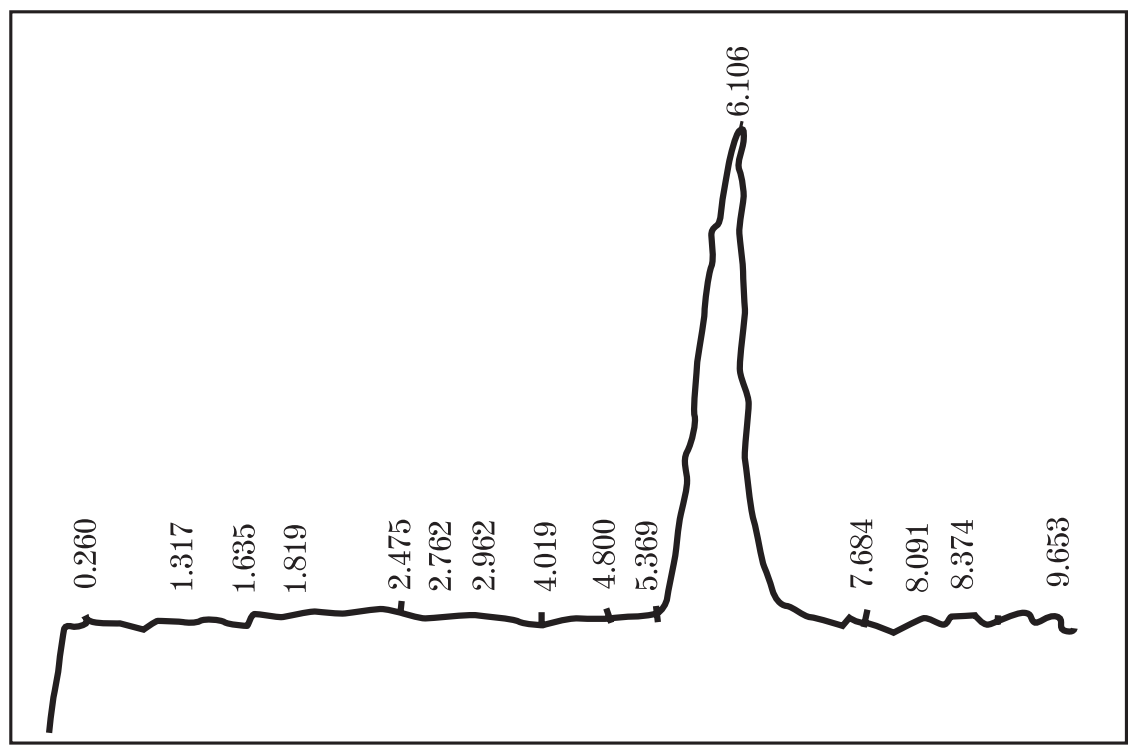

Ilustración 3. Cromatograma correspondiente al pico de OTA en los patrones

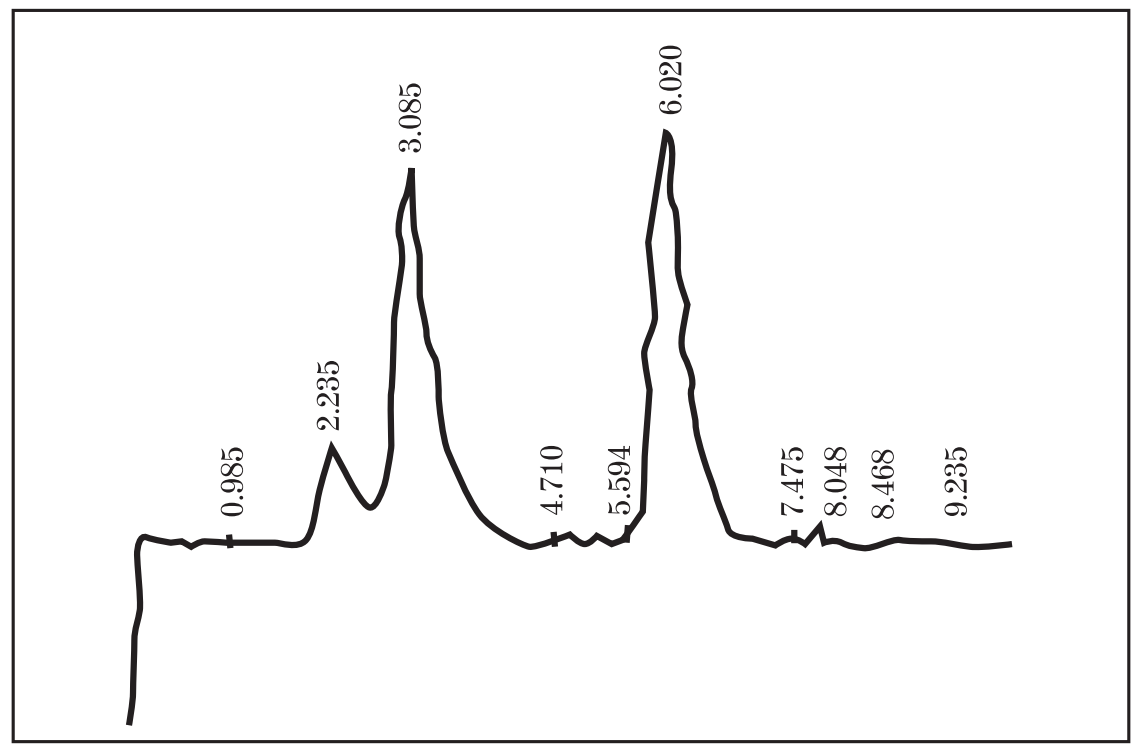

Ilustración 4. Cromatograma correspondiente al pico de OTA en las muestras analizadas 
Cuadro 2. Determinación de OTA vía HPLC para muestras procedentes de diferentes lugares de Nicaragua

\begin{tabular}{|c|c|c|c|c|c|c|c|c|}
\hline Origen de la muestra & $\begin{array}{c}\text { Tubo } \\
\#\end{array}$ & $\begin{array}{l}\text { \# de } \\
\text { Inj's }\end{array}$ & Area & DS & Conc. OTA & DS & $\begin{array}{c}\text { Rango } \\
\text { OTA }(\mu \mathrm{g} / \mathrm{L})\end{array}$ & DS \\
\hline $\begin{array}{l}\text { NATURALMENTE } \\
\text { CONTAMINADA }\end{array}$ & $\begin{array}{r}1 \\
2 \\
3 \\
-\end{array}$ & $\begin{array}{r}3 \\
3 \\
3 \\
-\end{array}$ & $\begin{array}{l}899962 \\
854386 \\
809136\end{array}$ & $\begin{array}{c}22240 \\
3824 \\
4764 \\
\end{array}$ & $\begin{array}{r}37.8 \\
35.9 \\
34.0\end{array}$ & $\begin{array}{l}0.35 \\
0.34 \\
0.33\end{array}$ & _ 35.9 & 1.91 \\
\hline Resumen Resultados & & 9 & 854495 & 40985 & & & 35.9 & 0.29 \\
\hline ISRAEL 1 & $\begin{array}{r}1 \\
2 \\
3 \\
-\end{array}$ & $\begin{array}{r}3 \\
3 \\
3 \\
-\end{array}$ & $\begin{array}{l}243500 \\
248755 \\
248625\end{array}$ & $\begin{array}{r}21890 \\
10634 \\
25750\end{array}$ & $\begin{array}{l}10.3 \\
10.5 \\
10.5\end{array}$ & $\begin{array}{l}0.26 \\
0.26 \\
0.26\end{array}$ & 10.4 & 0.13 \\
\hline Resumen Resultados & & 9 & 246960 & 17904 & & & 10.4 & 0.19 \\
\hline GUAPOTAL & $\begin{array}{r}1 \\
2 \\
3 \\
-\end{array}$ & $\begin{array}{r}3 \\
3 \\
3 \\
-\end{array}$ & $\begin{array}{l}436673 \\
442279 \\
451148\end{array}$ & $\begin{array}{c}8069 \\
37982 \\
18475 \\
\end{array}$ & $\begin{array}{r}18.4 \\
18.6 \\
19.0\end{array}$ & $\begin{array}{l}0.27 \\
0.27 \\
0.27\end{array}$ & 18.6 & 0.31 \\
\hline Resumen Resultados & & 9 & 443367 & 22410 & & & 18.6 & 0.20 \\
\hline $\begin{array}{l}\text { CANAVALIA } \\
\text { Resumen resultados }\end{array}$ & $\begin{array}{r}1 \\
2 \\
3 \\
-\end{array}$ & $\begin{array}{r}3 \\
3 \\
3 \\
-9 \\
\end{array}$ & $\begin{array}{c}0 \\
467 \\
804 \\
-423 \\
\end{array}$ & $\begin{array}{c}0 \\
808 \\
-1392 \\
877\end{array}$ & $\begin{array}{r}0.0 \\
0.0 \\
0.1 \\
-\end{array}$ & $\begin{array}{l}0.29 \\
0.29 \\
0.29\end{array}$ & $-\frac{0.0}{0.0}$ & $\begin{array}{r}0.02 \\
0.22\end{array}$ \\
\hline TUMA & $\begin{array}{r}1 \\
2 \\
3 \\
-\end{array}$ & $\begin{array}{r}3 \\
3 \\
3 \\
-\end{array}$ & $\begin{array}{l}44784 \\
30052 \\
37080\end{array}$ & $\begin{array}{l}24704 \\
13234 \\
10402\end{array}$ & $\begin{array}{l}1.9 \\
1.3 \\
1.6 \\
.\end{array}$ & $\begin{array}{l}0.28 \\
0.28 \\
0.28\end{array}$ & 1.6 & 0.31 \\
\hline Resumen Resultados & & 9 & 37305 & 16252 & & & 1.6 & 0.22 \\
\hline DALIA & $\begin{array}{r}1 \\
2 \\
3 \\
-\end{array}$ & $\begin{array}{r}3 \\
3 \\
3 \\
-\end{array}$ & $\begin{array}{r}45225 \\
36064 \\
22772 \\
-\end{array}$ & $\begin{array}{c}22889 \\
7199 \\
10474\end{array}$ & $\begin{array}{l}1.9 \\
1.5 \\
1.0\end{array}$ & $\begin{array}{l}0.28 \\
0.28 \\
0.29 \\
\end{array}$ & 1.5 & 0.47 \\
\hline Resumen Resultados & & 9 & 34687 & 16339 & & & 1.5 & 0.22 \\
\hline WASLALA & $\begin{array}{l}1 \\
2 \\
3\end{array}$ & $\begin{array}{l}3 \\
3 \\
3\end{array}$ & $\begin{array}{l}460 \\
5940 \\
3007\end{array}$ & $\begin{array}{l}4520 \\
4633 \\
1052\end{array}$ & $\begin{array}{l}0.2 \\
0.3 \\
0.1\end{array}$ & $\begin{array}{l}0.29 \\
0.29 \\
0.29 \\
\end{array}$ & 0.2 & 0.06 \\
\hline Resumen Resultados & & 9 & 4569 & 3519 & & & 0.2 & 0.22 \\
\hline Resumen Resultados & $\begin{array}{r}1 \\
2 \\
3 \\
-\end{array}$ & $\begin{array}{r}3 \\
3 \\
-3 \\
9\end{array}$ & $\begin{array}{r}2367 \\
6523 \\
1610 \\
-3500 \\
3500\end{array}$ & $\begin{array}{r}2381 \\
4118 \\
1427 \\
-3379 \\
\end{array}$ & $\begin{array}{l}0.1 \\
0.3 \\
0.1\end{array}$ & $\begin{array}{l}0.29 \\
0.29 \\
0.29 \\
-2\end{array}$ & $-\frac{0.2}{0.2}$ & $\begin{array}{r}0.11 \\
0.22\end{array}$ \\
\hline ISRAEL 2 & $\begin{array}{r}1 \\
2 \\
3 \\
-\end{array}$ & $\begin{array}{r}3 \\
3 \\
3 \\
-\end{array}$ & $\begin{array}{l}1695957 \\
1640108 \\
1694176\end{array}$ & $\begin{array}{l}13441 \\
21714 \\
10777\end{array}$ & $\begin{array}{r}71.3 \\
68.9 \\
71.2 \\
-\end{array}$ & $\begin{array}{l}0.61 \\
0.59 \\
0.61\end{array}$ & 70.5 & 1.33 \\
\hline Resumen Resultados & & $\overline{9}$ & $167674 \overline{7}$ & 30786 & & & 70.5 & 0.57 \\
\hline
\end{tabular}


Para comparar el método Vicam con el HPLC, se adicionaron cantidades de OTA a muestras de café a fin de valorar la concentración detectada en el Fluorímetro. El Cuadro 3 muestra la contracción de OTA añadida y la detectada:

Cuadro 3. Concentración añadida y detectada en el fluorímetro

\begin{tabular}{|c|c|}
\hline OTA Adicionada & OTA Detectada \\
\hline \multirow{2}{*}{$0 \quad \mathrm{ppb}$} & $0.0 \mathrm{ppb}$ \\
\cline { 2 - 2 } & $0.0 \mathrm{ppb}$ \\
\cline { 2 - 2 } & $0.0 \mathrm{ppb}$ \\
\hline \multirow{2}{*}{$1.0 \mathrm{ppb}$} & $1.0 \mathrm{ppb}$ \\
\cline { 2 - 2 } & $0.9 \mathrm{ppb}$ \\
\cline { 2 - 2 } & $1.1 \mathrm{ppb}$ \\
\hline \multirow{2}{*}{$2.0 \mathrm{ppb}$} & $1.7 \mathrm{ppb}$ \\
\cline { 2 - 2 } & $2.1 \mathrm{ppb}$ \\
\cline { 2 - 2 } & $1.9 \mathrm{ppb}$ \\
\hline \multirow{2}{*}{$4.0 \mathrm{ppb}$} & $3.1 \mathrm{ppb}$ \\
\hline \multirow{2}{*}{} & $4.1 \mathrm{ppb}$ \\
\cline { 2 - 2 } & $3.9 \mathrm{ppb}$ \\
\hline
\end{tabular}

Se procedió a determinar la concentración de OTA en muestras de café procedentes de diferentes lugares de Nicaragua, siguiendo el método Vicam Ochratest (ver Cuadro 4).

Cuadro 4. Concentración de OTA en muestras de diferentes sitios de Nicaragua

\begin{tabular}{|l|c|}
\hline Origen de la muestra & Concentración de OTA $[\mu \mathrm{g} / \mathrm{L}]$ \\
\hline ISRAEL 1 & 11.1 \\
\hline ISRAEL 2 & 71.2 \\
\hline CANAVALIA & $0 *$ \\
\hline GUAPOTAL & $19.0 * *$ \\
\hline TUMA & $2.0^{*}$ \\
\hline WASLALA & $0.5^{*}$ \\
\hline DALIA & $1.2^{*}$ \\
\hline RANCHO GRANDE & $0.4^{*}$ \\
\hline NATURALMENTE CONTAMINADA & 36.0 粆 \\
\hline
\end{tabular}

: Café vendido en grano en mercado capitalino

橉: Contaminado naturalmente

*: Valor en el rango recomendado por la Unión Europea

$*$ : Alto valor, probablemente debido a las condiciones de secado y almacenamiento 
En el Cuadro 4 se observa que las muestras señaladas con * tienen una concentración de OTA por debajo de tres ppb, máximo aceptado por la Unión Europea hasta fecha reciente.

\section{Resultados de la Validación del Método Analítico:}

\section{Limite de detección}

Para calcular el límite de detección, se prepararon soluciones de adición de OTA a niveles de cero, uno, dos y cuatro ppbs, a fin de medir la concentración detectada en el Fluorímetro.

Cuadro 5. Concentraciones adicionadas y detectadas de OTA para el cálculo del límite de detección

\begin{tabular}{|c|c|}
\hline OTA Adicionada & OTA Detectada \\
\hline \multirow{2}{*}{$0 \mathrm{ppb}$} & $0.0 \mathrm{ppb}$ \\
\cline { 2 - 2 } & $0.0 \mathrm{ppb}$ \\
\cline { 2 - 2 } & $0.0 \mathrm{ppb}$ \\
\hline \multirow{2}{*}{$1.0 \mathrm{ppb}$} & $1.0 \mathrm{ppb}$ \\
\cline { 2 - 2 } & $0.9 \mathrm{ppb}$ \\
\cline { 2 - 2 } & $1.1 \mathrm{ppb}$ \\
\hline \multirow{2}{*}{$2.0 \mathrm{ppb}$} & $1.7 \mathrm{ppb}$ \\
\cline { 2 - 2 } & $2.1 \mathrm{ppb}$ \\
\cline { 2 - 2 } & $1.9 \mathrm{ppb}$ \\
\hline \multirow{2}{*}{$4.0 \mathrm{ppb}$} & $3.1 \mathrm{ppb}$ \\
\cline { 2 - 2 } & $4.1 \mathrm{ppb}$ \\
\cline { 2 - 2 } & $3.9 \mathrm{ppb}$ \\
\hline
\end{tabular}

El limite de detección calculado para los valores del cuadro 5 es de 1 ppb

\section{Linealidad}

La linealidad se determinó usando Ocratoxina A adicionada a muestras de grano de café verde en un rango de 5 ppb a 50 ppb. (Ver Cuadro 6 e Ilustración 5).

Cuadro 6. Concentración de OTA adicionada y medida para valorar la linealidad

\begin{tabular}{|c|c|}
\hline OTA adicionada $[\mu \mathbf{g} / \mathbf{L}]$ & OTA medida $[\mu \mathbf{g} / \mathbf{L}]$ \\
\hline 0 & 0 \\
\hline 5 & 6 \\
\hline 10 & 12 \\
\hline 20 & 21 \\
\hline 50 & 53 \\
\hline
\end{tabular}




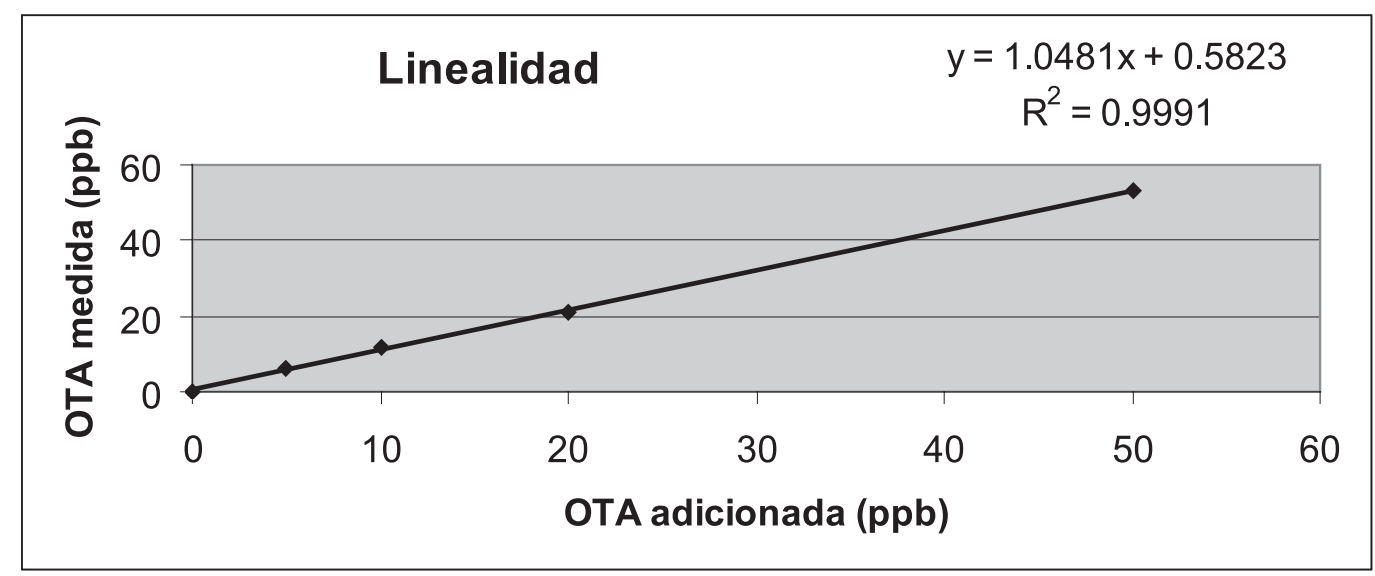

Ilustración 5. Linealidad para la concentración de OTA medida y adicionada

El coeficiente de correlación R de 0.9991 de la Ilustración 5 indica que la linealidad del método es muy buena.

\section{Exactitud}

La exactitud se mide por medio de la reproducibilidad y se siguió el mismo protocolo de extracción y purificación que la repetibilidad, variando únicamente el número de analistas, que en esta ocasión eran dos. El ensayo se realizó el mismo día y en la mismas condiciones experimentales. Para ello, se usaron muestras de café verde con OTA adicionada a los niveles de 5, 10, 20 y 50 ppb. El Cuadro 7 y la Ilustración 6 muestran los diferentes niveles de OTA adicionada y la linealidad del ensayo, respectivamente.

Cuadro 7. Ensayo para la reproducibilidad a diferentes valores de OTA adicionada

\begin{tabular}{|c|c|}
\hline OTA Adicionada (ppb) & OTA Detectada (ppb) \\
\hline 5 & 5.3 \\
\hline 10 & 11.5 \\
\hline 20 & 23 \\
\hline 50 & 53 \\
\hline
\end{tabular}




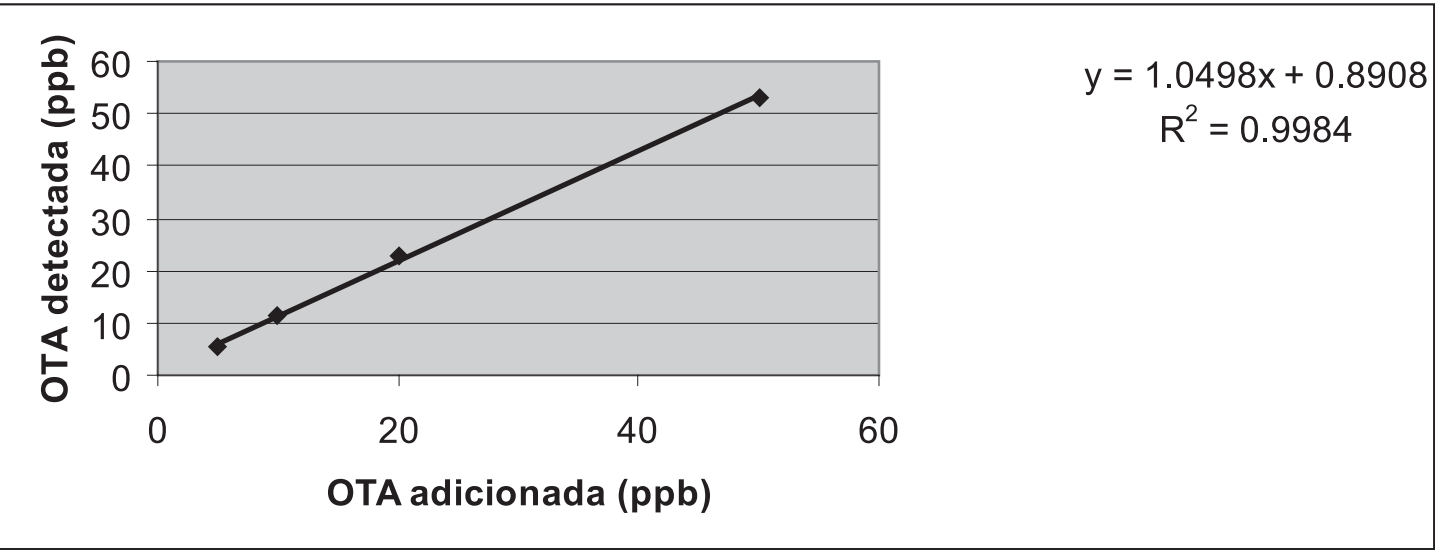

Ilustración 6. Linealidad de patrones en el ensayo de reproducibilidad

\section{Precisión}

La precisión expresada en términos de repetibilidad se establece analizando réplicas de una muestra adicionada con patrón de OTA en un mismo laboratorio en idénticas condiciones.

La precisión se determinó adicionando extractos de café a niveles de dos, 20 y 50 ppb. Se midieron diez réplicas de cada muestra adicionada y, con la media, se determinó la desviación estándar y el porcentaje de coeficiente de variación.

Cuadro 8. Coeficientes de variación para valorar la precisión a diferentes niveles de OTA adicionada

\begin{tabular}{|c|c|c|c|c|c|}
\hline $\begin{array}{c}\text { Nivel } \\
\text { adicionado } \\
(\mathbf{p p b})\end{array}$ & $\begin{array}{c}\text { Cantidad } \\
\text { detectada } \\
\mathbf{( p p b )}\end{array}$ & $\begin{array}{c}\text { Nivel adicionado } \\
\mathbf{( p p b )}\end{array}$ & $\begin{array}{c}\text { Cantidad } \\
\text { detectada } \\
\mathbf{( p p b})\end{array}$ & $\begin{array}{c}\text { Nivel } \\
\text { adicionado } \\
\mathbf{( p p b )}\end{array}$ & $\begin{array}{c}\text { Cantidad } \\
\text { detectada } \\
\mathbf{( p p b )}\end{array}$ \\
\hline 2 & 2.1 & 20 & 21 & 50 & 46 \\
\hline 2 & 1.6 & 20 & 18 & 50 & 52 \\
\hline 2 & 1.7 & 20 & 19 & 50 & 51 \\
\hline 2 & 2.2 & 20 & 22 & 50 & 45 \\
\hline 2 & 2.5 & 20 & 19 & 50 & 57 \\
\hline 2 & 2.1 & 20 & 21 & 50 & 45 \\
\hline 2 & 0.9 & 20 & 17 & 50 & 51 \\
\hline 2 & 2.8 & 20 & 21 & 50 & 45 \\
\hline 2 & 1.8 & 20 & 18 & 50 & 53 \\
\hline 2 & 3.0 & 20 & 22 & 50 & 52 \\
\hline Promedio & 2.1 & - & 19.8 & - & 49.7 \\
\hline $\begin{array}{c}\text { Desviación } \\
\text { estándar }\end{array}$ & 0.58 & - & 1.72 & - & 4.0 \\
\hline $\begin{array}{c}\text { \% Coeficiente } \\
\text { de Variación }\end{array}$ & 27.6 & - & 8.68 & - & 8.04 \\
\hline
\end{tabular}


Posteriormente, otro día, se adicionó OTA a diez réplicas de café verde, a un nivel de tres ppb y se obtuvieron los siguientes resultados:

Cuadro 9. Réplicas realizadas en las mismas condiciones y con el mismo equipo

\begin{tabular}{|c|c|}
\hline Nivel adicionado (ppb) & Cantidad detectada (ppb) \\
\hline 3 & 2.4 \\
\hline 3 & 2.6 \\
\hline 3 & 2.4 \\
\hline 3 & 2.5 \\
\hline 3 & 2.7 \\
\hline 3 & 2.6 \\
\hline 3 & 2.3 \\
\hline 3 & 2.3 \\
\hline 3 & 2.2 \\
\hline 3 & 2.5 \\
\hline & 2.4 \\
\hline Principal & $\mathbf{0 . 1 6}$ \\
\hline Desviación Estándard & $\mathbf{6 . 6 6}$ \\
\hline \%Coeficiente de Variación & \\
\hline
\end{tabular}

Este método muestra una buena precisión con un coeficiente de variación menor que 10\% a través del rango de tres a $50 \mathrm{ppb}$.

\section{Porcentaje de recuperación}

La recuperación se obtiene mediante el método de adiciones estándar. La muestra se analiza adicionada a niveles distintos, para evaluar el porcentaje de recuperación.

Cuadro 10. Porcentaje de recuperación a diferentes niveles de OTA

\begin{tabular}{|c|c|c|}
\hline OTA Adicionada (ppb) & OTA Detectada (ppb) & \% Recuperación \\
\hline 0.25 & 0.21 & 84.0 \\
\hline 0.50 & 0.37 & 74.0 \\
\hline 1.0 & 0.94 & 94.0 \\
\hline 2.0 & 1.70 & 85.0 \\
\hline 4.0 & 2.85 & 71.0 \\
\hline 10 & 7.4 & 74.0 \\
\hline 30 & 22.0 & 73.3 \\
\hline 50 & 35.5 & 71.0 \\
\hline
\end{tabular}


El Cuadro 10 muestra el porcentaje de recuperación en el rango 0.25 a 50 ppb es, aproximadamente, de 78.3\% \pm 10.7 . Este porcentaje está dentro del margen aceptado (60$115 \%)$.

\section{Correlación entre Fluorímetro (Vicam Ochratest) y método HPLC}

Considerando que los resultados obtenidos por el método rápido Ochratest son tan precisos, exactos y reproducibles como el HPLC, se procedió a comparar ambos métodos a fin de obtener, mediante el análisis de regresión lineal, el coeficiente de correlación. Para ello, se tomaron 24 muestras (nueve no adicionadas y 15 adicionadas). El Cuadro 11 presenta los resultados de ese estudio.

Cuadro 11. Correlación entre Vicam Ochratest y HPLC para muestras adicionadas y no adicionadas con OTA

\begin{tabular}{|c|c|c|c|}
\hline Muestra & Nivel adicionado & Fluorímetro & $\begin{array}{c}\text { HPLC(valores corregidos para la } \\
\text { recuperación) }\end{array}$ \\
\hline \multirow[t]{3}{*}{1} & 0 & 0.23 & 0 \\
\hline & 5 & 4.8 & 5.2 \\
\hline & 20 & 23 & 22 \\
\hline \multirow[t]{3}{*}{2} & 0 & 0.62 & 0 \\
\hline & 5 & 4.1 & 4.8 \\
\hline & 20 & 19 & 18.6 \\
\hline \multirow[t]{3}{*}{3} & 0 & 0.63 & 0 \\
\hline & 5 & 4.9 & 5.5 \\
\hline & 20 & 24 & 21 \\
\hline 4 & 0 & 1.5 & 0.4 \\
\hline 5 & 0 & 0.8 & 0 \\
\hline 6 & 0 & 0.5 & 0 \\
\hline \multirow[t]{4}{*}{7} & 0 & 0.65 & 0 \\
\hline & 10 & 13.6 & 12 \\
\hline & 30 & 29 & 28 \\
\hline & 50 & 54 & 53 \\
\hline \multirow[t]{4}{*}{8} & 0 & 0.52 & 0 \\
\hline & 10 & 10 & 10.3 \\
\hline & 30 & 27 & 10.2 \\
\hline & 50 & 53 & 51.4 \\
\hline \multirow[t]{4}{*}{9} & 0 & 0.3 & 0 \\
\hline & 10 & 10 & 10 \\
\hline & 30 & 26 & 24 \\
\hline & 50 & 48 & 42 \\
\hline
\end{tabular}


Los resultados muestran una buena correlación entre los dos diferentes métodos. Cuando fueron analizados por regresión lineal, los resultados mostraron un r de 0.998.

\section{Discusión}

Se han comparado tres métodos para la determinación de OTA en café (TLC, HPLC-Columnas de inmunoafinidad y el test rápido Vicam Ochratest. La determinación cromatográfica por el método TLC no es un método óptimo para la determinación de OTA en café por ser lento, cualitativo y caro en reactivos contaminantes. Los resultados obtenidos por el test rápido Vicam Ochratest son comparables con los alcanzados con HPLC, método sensible y reproducible. Tomando en cuenta esta consideración, se validó el test rápido Vicam Ochratest, concluyendo que el método es lineal en el margen de cinco a $50 \mu \mathrm{g} / \mathrm{L}$; la exactitud del método, expresada en porcentaje de recuperación, es del 78.3\% \pm 10.7 ; la repetibilidad, para muestras de café adicionadas con patrón de OTA al nivel de $3 \mu \mathrm{g} / \mathrm{kg}$ por muestra, expresada en coeficientes de variación, es de $2.4 \mu \mathrm{g} / \mathrm{kg} \pm 6.6 \%$.

El test rápido Vicam Ochratest y el método HPLC tienen una buena correlación, indicada por regresión lineal $(\mathrm{r}=0.998)$. El método se aplicó a nueve $(9)$ muestras procedentes de diferentes lugares de Nicaragua, encontrándose en cuatro de ellas concentraciones superiores a las aceptadas por la Unión Europea.

Este trabajo está orientado a promover tecnología apropiada y de bajo costo, accesible a pequeños caficultores asociados en cooperativas, a fin de contribuir a la mejora de la calidad del café y del precio en el mercado internacional. Una vez que el método validado haya sido revalidado a través del resultado de análisis de otros lugares de Nicaragua, se pretende solicitar su acreditación ante el Organismo Nacional de Acreditación del Ministerio y Fomento de Industrias de Nicaragua para OTA en granos de café verde.

\section{Agradecimientos}

Esta investigación contó con el apoyo de la Dirección de Investigación y Proyección Social de la UCA, a través de los Fondos de Investigación (FIUCA); y de los Doctores Susan y Charles Jackels, de la Universidad de Seattle y Washington-Bothel.

\section{Referencias bibliográficas}

- BATISTA, L. R., CHALFOUN, S. M., PRADO G. et al., (2003). "Toxigenic fungi associated with processed (green) coffee beans (Coffea Arabica L.)”. International Journal of Food Microbiology, 85, pág. 293-300. USA.

- CASTRO, C., PUJOL, M., MARTÍ F. et al., (2000). Validación de métodos analíticos, A.E.F.I, sección catalana. Comisión de normas de buena fabricación y control de calidad, Biblioteca de la Universidad de Barcelona, España

- DOS SANTOS, E., PITTET, A. y VARGAS, E. A. (2004). Determination of Ochratoxin A in green Coffee by Immunoaffinity Column Cleanup and Liquid Chromatography, Collaborative Study, Nestle Research Center, Switzerland 
- FRANK, M. (1999). Prevención contra las micotoxinas y descontaminación. Sistema de HACCP y su potencial para combatir las micotoxinas: Una evaluación de la Ocratoxina A en la producción del café. Tercera Conferencia internacional FAO/OMS/PNUMA sobre micotoxinas, Túnez.

- PITTET, A. y DELPHINE, R. (2002). "Rapid, Low Cost Thin-Layer Chromatographic Screening Method for the detection of Ochratoxin A in Green Coffee at a Control Level of 10 $\mu \mathrm{g} / \mathrm{kg}$. J. Agrir. Food Chem., 50(2), pág. 243-247. USA.

- VENTURA, M. y VALLEJOS, C., et al., (2003). "Analysis of Ochratoxin A in coffee by solid-phase cleanup and narrow-bore liquid chromatography-fluorescence detector-mass spectrometr". Journal of Agricultural and Food Chemistry, 51, pág. 7564 -7567.USA.

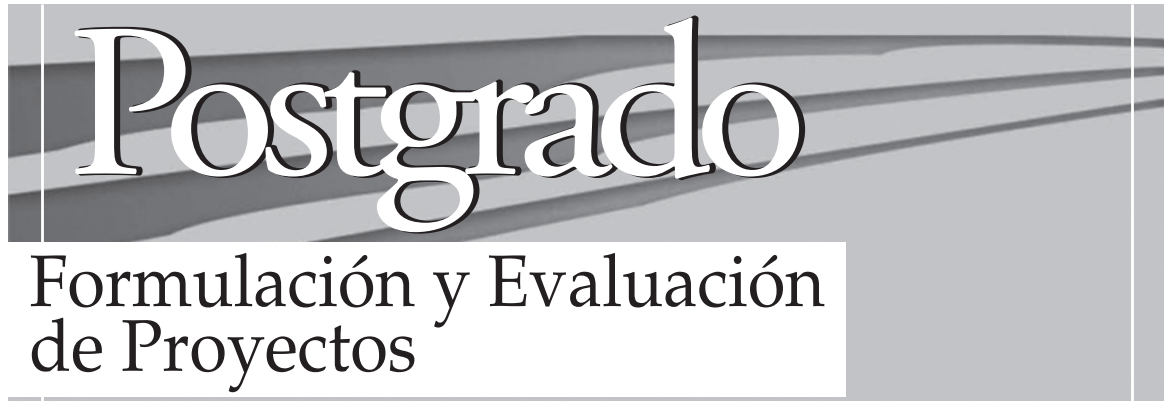

Dirigido a:

Profesionales que laboran en el sector público y privado en las áreas de formulación, evaluación, gestión y administración de proyectos. Igualmente profesionales que laboran en organismos no gubernamentales e internacionales que demandan capacitación en el tema de los proyectos de inversión.

\section{Plan de estudios:}

- Análisis Microeconómico.

- Análisis Financiero.

- Identificación de Proyectos.

- Formulación de Proyectos.

- Evaluación Financiera de Proyectos.

- Evaluación Eco y Social de Proyectos.

\section{Modalidad y horario:}

Modalidad regular. 5 meses.

Lunes a Jueves de 6:00 a 9:00 p.m.

Modalidad sabatina. 7 meses.

De 8:00 a.m. a 4:00 p.m.

\section{Inicio:}

Regular: 6 marzo, 2006

Sabatino: 11 marzo, 2006

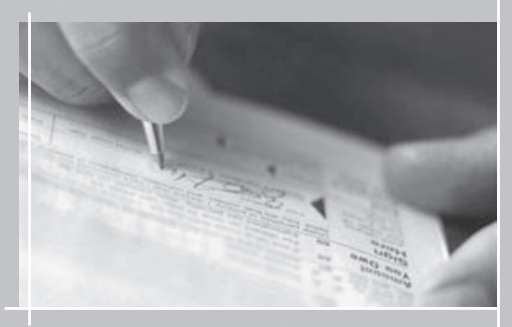

Msc. Ernesto Pérez Delgado, Coordinador del área de Postgrado y Maestría en Economía Facultad de Ciencias Económicas y Empresariales

Tel:278 39292788185 / Fax:270 3627 / E-mail:ernesto@ns.uca.edu.ni / www.uca.edu.ni 